\title{
Cabozantinib for the Treatment of Advanced Hepatocellular Carcinoma: Current Data and Future Perspectives
}

\author{
Jörg Trojan ${ }^{1}$ (D) \\ Published online: 15 July 2020 \\ (c) The Author(s) 2020
}

\begin{abstract}
Cabozantinib (Cabometyx $\left.{ }^{\circledR}\right)$ is a potent multikinase inhibitor targeting the vascular endothelial growth factor (VEGF) receptor 2 , the mesenchymal-epithelial transition factor (MET) receptor, and the "anexelekto" (AXL) receptor tyrosine kinase. It is approved for the treatment of advanced hepatocellular carcinoma (HCC) after failure of sorafenib in Europe (since November 2018) and in the USA (since January 2019). The approval of cabozantinib was based on results of the randomized, placebocontrolled, phase 3 CELESTIAL trial in patients with unresectable HCC, who received one or two prior lines of treatment including sorafenib. At the second planned interim analysis, the trial was stopped, because the primary end point overall survival was clearly in favor for cabozantinib. Additionally, median progression-free survival was superior to placebo. The most common $\geq$ grade 3 relevant adverse events in patients with HCC treated with cabozantinib were palmar-plantar erythrodysesthesia, hypertension, fatigue, and diarrhea. In this review, current data on cabozantinib for the treatment of patients with advanced HCC, with a focus on the management of common adverse events and ongoing clinical trials, are discussed.
\end{abstract}

\section{Key Points}

Cabozantinib is approved for patients with sorafenibpretreated advanced hepatocellular carcinoma (HCC).

The management of hypertension and gastrointestinal and dermatological toxicity is crucial to maximize the benefit of this targeted therapy in HCC.

Cabozantinib is currently being further developed in combination with immune checkpoint inhibitors in patients with advanced HCC.

\section{Introduction}

Liver cancer is the fourth most frequent cause of cancerrelated mortality, with 782,000 deaths in 2018 worldwide [1]. The most common form of liver cancer is hepatocellular

Jörg Trojan

trojan@em.uni-frankfurt.de

1 Universitätsklinikum Frankfurt, Medizinische Klinik 1, Theodor-Stern-Kai 7, 60590 Frankfurt am Main, Germany carcinoma (HCC), with over 600,000 new cases annually. Most cases of HCC occur in Asia and sub-Saharan Africa due to endemic chronic hepatitis B virus infection. In Western countries, alcohol and chronic hepatitis $C$ virus infection are the predominant risk factors, and due to rising prevalence of non-alcoholic steatohepatitis, the incidence of HCC is steadily rising. Most patients with HCC have underlying liver cirrhosis, which not only limits surgical options, but also might result in different safety profiles of systemic agents. Despite new therapeutic options, HCC is an aggressive condition and patients with advanced disease have a poor prognosis [2].

The landscape of systemic therapy for advanced HCC has been evolving rapidly since 2017 . For 10 years, the multi-targeted antiangiogenic tyrosine kinase inhibitor sorafenib was the only approved systemic therapy $[3,4]$. After 2017, the multi-targeted tyrosine kinase inhibitor regorafenib (for patients who tolerated sorafenib), the predominantly anti-angiogenic tyrosine kinase lenvatinib (as alternative first-line treatment), and the vascular endothelial growth factor (VEGF) receptor 2 (VEGFR2)-directed monoclonal antibody ramucirumab (for patients with alpha fetoprotein (AFP) $\geq 400 \mathrm{ng} / \mathrm{mL}$ ), all targeting the VEGF pathway, were approved as further systemic treatment options [5-7]. In the USA, the programmed death receptor 1 (PD-1) immune checkpoint inhibitors nivolumab and pembrolizumab are approved after sorafenib-failure, based on 
two non-randomized phase 2 trials [8,9]. Just recently, the first immunotherapy combination of the anti-programmed death-ligand 1 (anti-PD-L1) antibody atezolizumab and the VEGF-A antibody bevacizumab was approved as first-line treatment based on the impressive results of the IMbrave150 study [10]. In contrast to other multi-tyrosine kinase inhibitors, cabozantinib targets in addition to VEGFR2 the mesenchymal-epithelial transition factor (MET) receptor and the "anexelekto" (AXL) receptor tyrosine kinase. All three are induced by hypoxia, and MET and AXL are involved in resistance to antiangiogenic therapy [11]. MET is a promising target in HCC, since the MET/HGF pathway is involved in HCC progression by promoting cellular proliferation, survival and invasion [12]. Currently, selective MET inhibitors, e.g., tepotinib and capmatinib, are in clinical development in HCC [13]. Capmatinib was recently approved by the Food and Drug Administration (FDA) for treatment of patients with non-small cell lung cancer (NSCLC), with specific mutations leading to mesenchymal-epithelial transition or MET exon 14 skipping [14]. Tivantinib, a selective MET inhibitor with cytotoxic properties, is not active in advanced HCC [15].

In November 2018, cabozantinib was approved in Europe and, subsequently, in January 2019, in the USA, based on the positive results of the worldwide randomized, placebo-controlled, phase 3 CELESTIAL trial in patients with unresectable HCC, who received one or two prior lines of treatment including sorafenib [16].

In this review, data on the use of cabozantinib in advanced HCC will be discussed, with special focus on side effects and their management in HCC, followed by the outlook of the ongoing development program of this drug in HCC.

\section{Pharmacological Properties}

Cabozantinib is a potent multi-targeted tyrosine kinase inhibitor of VEGFR2 (half maximal inhibitory concentration [ $\mathrm{IC}_{50}$ ] $0.035 \mathrm{nmol} / \mathrm{L}$ ), the hepatocyte growth factor receptor MET $\left(\mathrm{IC}_{50} 1.3 \mathrm{nmol} / \mathrm{L}\right)$, AXL ( $\left.\mathrm{IC}_{50} 7 \mathrm{nmol} / \mathrm{L}\right)$, fms like tyrosine kinase 3 (FLT3) $\left(\mathrm{IC}_{50} 11.3 \mathrm{nmol} / \mathrm{L}\right), \mathrm{KIT}\left(\mathrm{IC}_{50} 4.6 \mathrm{nmol} / \mathrm{L}\right)$, RET ( $\left(\mathrm{IC}_{50} 5.2 \mathrm{nmol} / \mathrm{L}\right)$, TIE-2 ( $\left.\mathrm{IC}_{50} 14.3 \mathrm{nmol} / \mathrm{L}\right)$, and RON $\left(\mathrm{IC}_{50} 124 \mathrm{nmol} / \mathrm{L}\right)$ [17]. The kinase inhibitor selectivity of cabozantinib differs from that of lenvatinib, sorafenib, and regorafenib (Table 1) [18-20]. Cabozantinib exhibited doserelated tumor growth inhibition, tumor regression, and/or metastasis inhibition in a broad range of preclinical tumor models. It is available as tablets (Cabometyx) and capsules (Cometriq, indicated for patients with unresectable locally advanced or metastatic medullary thyroid carcinoma), which are not bioequivalent. Cabometyx is indicated as monotherapy for patients with advanced HCC after failure of sorafenib and for patients with advanced renal cell carcinoma (RCC)
Table 1 Target inhibition of cabozantinib, lenvatinib, sorafenib, and regorafenib [15-18]

\begin{tabular}{lllll}
\hline & \multicolumn{3}{l}{$\mathrm{IC}_{50}(\mathrm{nmol} / \mathrm{L})$} & \\
\cline { 2 - 5 } & Cabozantinib & Lenvatinib & Sorafenib & Regorafenib \\
\hline VEGFR2 & 0.035 & 4 & 90 & 4.2 \\
VEGFR1 & - & 22 & - & 13 \\
VEGFR3 & - & 5.2 & 20 & 46 \\
MET & 1.3 & - & - & - \\
AXL & 7 & - & - & - \\
FLT3 & 11.3 & - & 58 & - \\
KIT & 4.6 & 100 & 68 & 7 \\
RET & 5.2 & - & - & 1.5 \\
TIE-2 & 14.3 & - & - & 311 \\
RON & 124 & - & - & - \\
FGFR1 & - & 46 & 580 & 202 \\
PDGFR $\alpha$ & - & 51 & - & - \\
PDGFR $\beta$ & - & 39 & 57 & 22 \\
EGFR & - & 6500 & - & - \\
RAF1 & - & - & 6 & 2.5 \\
BRAF WT & - & - & 22 & 28 \\
BRAF V600E & - & - & 38 & 19 \\
\hline
\end{tabular}

$A X L$ anexelekto, EGFR epidermal growth factor receptor, $F G F R$ fibroblast growth factor receptor, FLT3 fms like tyrosine kinase 3, $I C_{50}$ half maximal inhibitory concentration, $M E T$ mesenchymal-epithelial transition factor, $P D G F R$ platelet-derived growth factor receptor, $V E G F R$ vascular endothelial growth factor receptor

as first-line therapy (intermediate or poor risk) or after failure of VEGF-targeted therapy [21, 22]. Based on preclinical data, cabozantinib is a promising agent for inhibiting tumor angiogenesis and metastasis in cancers with dysregulated VEGFR, MET, and AXL signaling [17]. Both MET and AXL are involved in resistance to anti-angiogenic therapy in RCC [11]. Since increased MET expression is a negative prognostic marker in HCC and upregulation was demonstrated after previous sorafenib treatment $[23,24]$, cabozantinib was further developed in this population.

\section{Therapeutic Efficacy in HCC}

\subsection{Phase 2 Data}

Cabozantinib was initially studied in a multi-cohort, randomized, discontinuation trial enrolling 526 patients with advanced tumors. Patients with nine different tumors, including HCC, were treated with cabozantinib capsules (100 mg once daily) [25]. In this trial, disease control rates (DCRs) $>40 \%$ were observed for castration-resistant prostate cancer, ovarian cancer, melanoma, metastatic breast cancer, HCC, and NSCLC. The HCC cohort $(n=41)$ was 
published separately in more detail [26]. This cohort recruited patients with advanced HCC from Europe or the USA $(n=26)$ and Asia $(n=15)$ with preserved liver function (Child-Pugh stage A only). The majority of patients received one line of prior systemic treatment $(n=30)$, which was sorafenib $(n=22)$ in the majority of patients. In total, nine patients were systemic treatment-naïve and two had received two prior lines of systemic therapy. The overall DCR at 12 weeks was $66 \%$, with a partial response in two patients (5\%). In Asian patients, the DCR was $73 \%$. The median progression-free survival (PFS) and overall survival (OS) were 5.2 and 11.5 months, respectively. The observed effects were independent of prior sorafenib therapy.

\subsection{Phase 3 Data}

Cabozantinib was evaluated as compared with placebo in previously treated patients with advanced HCC in a global, randomized, double-blind, phase 3 trial (CELESTIAL, XL184-309) [16]. A total of 707 patients with preserved liver function (Child-Pugh stage A) were randomly assigned in a 2:1 ratio to receive cabozantinib tablets (60 mg once daily) or matching placebo. Eligible patients had received previous treatment with sorafenib, had disease progression after at least one systemic treatment for HCC, and may have received up to two previous systemic regimens for advanced HCC. The primary end point was OS. Secondary end points were PFS and the objective response rate (ORR). At the second planned interim analysis, the trial showed significantly longer OS in the cabozantinib arm. Median OS was 10.2 months versus 8.0 months with placebo (hazard ratio [HR] 0.76; $95 \%$ confidence interval [CI] 0.63-0.92; $p=0.005)$. Median PFS was 5.2 months versus 1.9 months with placebo (HR $0.44 ; 95 \%$ CI $0.36-0.52 ; p<0.001)$, and the ORRs were $4 \%$ and less than $1 \%$, respectively $(p=0.009)$. Of interest, the lowest HR both for death and PFS was seen in patients with chronic hepatitis B virus infection as the underlying etiology of liver disease. In comparison with other second-line treatments, this is an argument for cabozantinib. Moreover, the HR for OS was better for patients in the second-line setting compared to patients in the third-line setting.

Since patients with Child-Pugh stage B liver cirrhosis were excluded in the CELESTIAL trial, further studies in patients with moderately impaired liver function (e.g., Child-Pugh stage B7 or 8) are needed [14]. But even in patients with Child-Pugh stage A, a further prognostic stratification using the albumin-bilirubin (ALBI) score, eliminating the subjective variables ascites and encephalopathy, is possible [27]. Accordingly, in patients with ALBI grade
1, median OS was 17.5 months with cabozantinib versus 11.4 months with placebo (HR 0.63; 95\% CI 0.46-0.86). In patients with ALBI grade 2, median OS was 8.0 months with cabozantinib versus 6.4 months with placebo (HR 0.84; 95\% CI 0.66-1.06). In patients with ALBI grade 1, median PFS was 6.5 months with cabozantinib versus 1.9 months with placebo (HR 0.42; 95\% CI 0.32-0.56), while in patients with ALBI grade 2, median PFS was 3.7 months with cabozantinib versus 1.9 months with placebo (HR 0.46 ; $95 \%$ CI 0.37-0.58) [28].

\subsection{Health-Related Quality of Life in CELESTIAL}

Both chronic liver disease including cirrhosis and HCC are associated with compromised health-related quality of life (HRQOL), and this is included as an important secondary endpoint in current pivotal trials in HCC [29]. In the CELESTIAL trial, the incremental quality-adjusted life years (QALYs) were reported from a post hoc analysis. Patient-reported health utilities were captured at each study visit using the EQ-5D-5L quality-of-life questionnaire. At day 50 of treatment, a minor reduction in mean total QALYs versus placebo was evident. However, with continued treatment, health utility increased in cabozantinib-treated patients, and at the end of the study, there was a clinically and statistically meaningful benefit in mean QALYs in favor of cabozantinib [30].

\subsection{Real-World Data of Cabozantinib in HCC}

Up to now, only a single retrospective cohort study of patients with advanced HCC receiving cabozantinib outside a clinical trial has been reported [31]. In this German/ Austrian study, 74 patients were included. Barcelona Clinic Liver Cancer (BCLC) stage C was present in $86 \%$ and Child-Pugh stage A cirrhosis in only $58 \%$ of patients. The median starting dose was $40 \mathrm{mg}$ once a day (QD). A partial response was documented in $5 \%$ of patients, the median treatment duration was 4.4 months, and the median OS was 7.7 months.

\section{Adverse Events of Cabozantinib in HCC}

Adverse events (AEs) caused by cabozantinib are typical for a variety of anti-angiogenic multi-targeted tyrosine kinase inhibitors [32]. In addition to hypertension, most of the current anti-angiogenic kinase inhibitors result in some kind of gastrointestinal and dermatological toxicity. Gastrointestinal AEs, such as diarrhea and mucositis, might ultimately result in weight loss and fatigue. The underlying mechanisms are still under investigation. Inhibition of platelet-derived 
growth factor receptors (PDGFRs) and c-Kit in the eccrine glands of the dermis and epidermis of the palms has been suggested as a mechanism contributing to palmar-plantar erythrodysesthesia (PPE) [33]. Moreover, hypertension might result from inhibition of VEGF-mediated nitric oxide synthase upregulation, which might lead to vasomotor dysfunction and degeneration of small blood vessels [32, 34]. Since VEGF and VEGFRs are also expressed in intestinal endothelial cells and c-Kit is important for the function of interstitial cells of cajal in the gastrointestinal tract, inhibition may cause gastrointestinal AEs [35, 36].

In a phase 2 , multi-cohort, randomized, discontinuation trial, 41 patients with advanced $\mathrm{HCC}$ were treated with cabozantinib capsules (100 mg QD) [26]. All patients reported any $\mathrm{AE}$ of any grade, whereas $85 \%$ of patients reported AEs $\geq$ grade 3 . The most common AEs were diarrhea (any grade $63 \%$; $\geq$ grade $320 \%$ ), PPE (56\%; 15\%), fatigue $(63 \% ; 20 \%)$, thrombocytopenia $(37 \% ; 15 \%)$, nausea $(37 \% ; 2 \%)$, vomiting $(37 \% ; 2 \%)$, decreased appetite $(29 \%$; $0 \%)$, aspartate aminotransferase (AST) increase $(27 \% ; 10 \%)$, hypertension $(24 \%$; $10 \%)$, rash $(24 \%$; $0 \%)$, asthenia $(22 \%$; $7 \%)$, weight loss $(22 \% ; 2 \%)$, constipation $(22 \% ; 0 \%)$, and hair color changes $(22 \%$; $0 \%)$ [25]. For the CELESTIAL phase 3 pivotal study, the formulation of cabozantinib was changed to tablets (60 mg QD). Treatment interruptions and dose reductions (step 1: $40 \mathrm{mg}$ QD; step 2: $20 \mathrm{mg} \mathrm{QD}$ ) were used to manage AEs [16]. Grade 3 or 4 AEs occurred in $68 \%$ of patients in the cabozantinib group and in $36 \%$ in the placebo group. Frequent $\geq$ grade 3 events were PPE (17\% with cabozantinib vs. $0 \%$ with placebo), hypertension $(16 \%$ vs. $2 \%$ ), AST increase (12\% vs. $7 \%$ ), fatigue (10\% vs. $4 \%$ ), and diarrhea (10\% vs. $2 \%$ ) (Fig. 1).

In the CELESTIAL trial, PPE and hypertension during cabozantinib treatment were shown to correlate with the outcome in a post hoc analysis [37]. Patients who experienced any grade PPE $(n=188)$ survived longer than patients without PPE $(n=282)(14.4$ months vs. 8.4 months; HR 0.59 ; 95\% CI 0.47-0.74). The same was shown for patients who experienced hypertension $\geq$ grade $3(n=61)$. The median OS was 16.1 months versus 9.5 months for those without treatment-related hypertension $\geq$ grade 3 (HR 0.56 ; 95\% CI 0.39-0.80).

In a real-world cohort, the most common AEs were fatigue and diarrhea [31].

\section{Management of Common Cabozantinib-Related Adverse Events}

Management of AEs is important for maintenance of quality of life, but also to assure treatment continuation, which might result in improved survival with cabozantinib. The most common AEs (all grades) reported in cabozantinibtreated HCC patients in the CELESTIAL trial were diarrhea, (54\%) decreased appetite (48\%), PPE (46\%), fatigue $(45 \%)$, nausea $(31 \%)$, vomiting (29\%), hypertension $(26 \%)$, liver enzyme increase (AST 22\% and alanine transaminase [ALT] 17\%), asthenia (22\%), dysphonia (19\%), abdominal pain $(18 \%)$, weight loss (17\%), mucosal inflammation (14\%) or stomatitis (13\%), rash (12\%), dysgeusia (12\%), hypoalbuminemia (12\%), thrombocytopenia (11\%), and dyspepsia (10\%) (Fig. 1) [16]. Of interest, AEs (all grades) seem to occur less frequently in HCC compared to RCC patients treated with cabozantinib, e.g., diarrhea $(72-75 \%)$, fatigue (59-86\%), hypertension (37-81\%), nausea (32-53\%), PPE $42-43 \%)$, vomiting (34\%), weight loss (32-35\%), stomatitis (22-36\%), and dysphonia (22\%) [38].

\subsection{Gastrointestinal Adverse Events}

Cabozantinib treatment in patients with $\mathrm{HCC}$ frequently causes gastrointestinal AEs with diarrhea (54\%; grade $\geq 3: 11 \%$ ) as the most common symptom. Moreover, diarrhea might be accompanied by nausea, vomiting, decreased appetite, abdominal pain, dysgeusia, and dyspepsia. However, these symptoms might also occur without diarrhea. Before cabozantinib initiation, it is crucial to inform patients about the possibility of developing diarrhea and, if this occurs, to encourage them to maintain hydration, avoid nicotine, and modify their diet (e.g., consume small and frequent meals and avoid caffeine, alcohol, lactose-containing products, and spicy, high-fiber, and fatty foods). In patients with higher grade diarrhea, self-administration of antidiarrheal medications (e.g., loperamide, starting dose $4 \mathrm{mg}$ then $2 \mathrm{mg}$ every $4 \mathrm{~h}$, or after each loose stool until the desired effect is achieved) should be initiated. For patients who frequently experience diarrhea, loperamide may also be taken preemptively, e.g., 30 min before cabozantinib intake. Moreover, replacement of fluids and electrolytes should be initiated as soon as possible. In the case of nausea and/or vomiting, chocolate, caffeine, alcohol, and nicotine should be strictly avoided and treatment with antiemetics (e.g., metoclopramide) should be initiated. Good oral hygiene is a prophylactic measure for the control of stomatitis, and mouthwashes with mixed actions (e.g., chamomile, dexapanthenol, xylocain, and/ or chlorhexidine) can be used for treatment. To overcome weight loss, a high-calorie diet and, if needed, dietary supplements are recommended $[32,38]$. Although cabozantinib absorption is acid dependent and therefore patients should not eat for at least $2 \mathrm{~h}$ before and at least $1 \mathrm{~h}$ after administration, concomitant use of the proton pump inhibitor esomeprazole had no effect on cabozantinib plasma exposure [39]. 
Fig. 1 Adverse events (AEs) (all grades and $\geq$ grade 3 ) in patients (percentage) treated with cabozantinib or placebo inside the CELESTIAL phase 3 trial. Listed are adverse events, regardless of causality, that were reported in at least $10 \%$ of patients in either group. Severity was graded according to National Cancer Institute Common Terminology Criteria for Adverse Events, version 4.0 [14]. Of note, the following AEs reported in CELESTIAL were omitted: bilirubin increase, anemia, back pain, insomnia, dizziness, headache, ascites, dyspnea, peripheral edema, cough, upper abdominal pain, pyrexia, and constipation. The absolute difference of occurrence of these AEs was less than 5\%, and therefore they were not considered to be cabozantinib related. $A L T$ alanine transaminase, $A S T$ aspartate aminotransferase, $P P E$ palmarplantar erythrodysesthesia

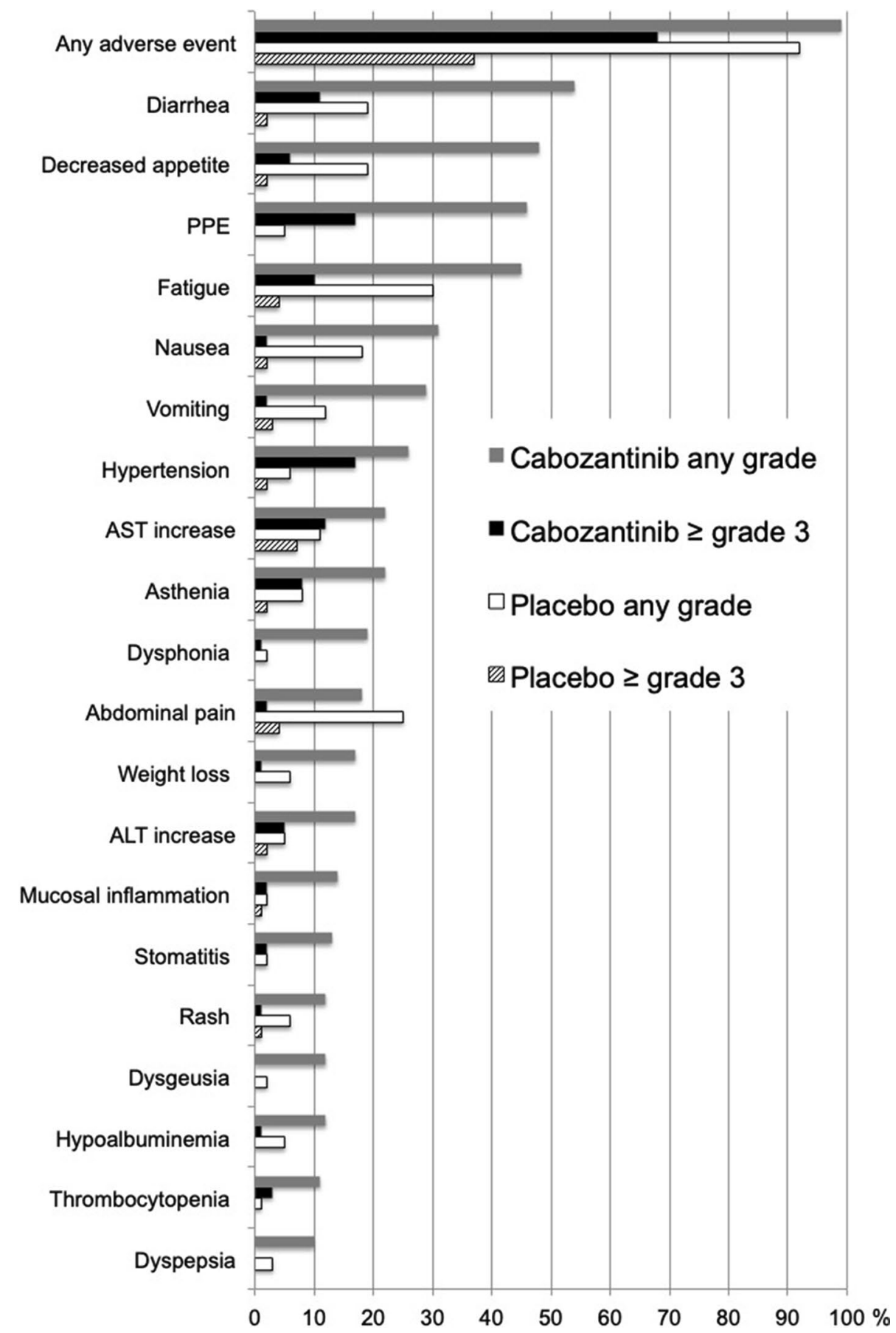

\subsection{Dermatological Adverse Events}

PPE is the most frequent dermatological adverse event, occurring in $46 \%$ (grade $\geq 3: 17 \%$ ) of HCC patients in the CELESTIAL trial, and often requires dose reductions or interruption, especially with higher grade PPE. Patients must be educated about prophylactic measures, which include urea-containing creams, pedicure to remove hyperkeratosis, topical exfoliation, and protection of pressure-sensitive areas before cabozantinib initiation (e.g., use of thick cotton gloves and socks). Adequate interventions are required to prevent worsening of skin symptoms such as blisters, desquamations, ulcerations, or necrosis of affected areas. Aggressive management of symptoms is recommended, including early dermatology referral. For grade 1 and 2 PPE, urea- and clobetasol-containing creams and analgesics if pain control is needed may be sufficient $[32,38]$. To prevent rash, the prophylactic use of hypoallergenic moisturizing 
creams, ointments for dry skin, and sunscreen (protection factor $\geq 30$ ) is recommended [22].

\subsection{Hypertension}

Hypertension is a common class effect of anti-angiogenic, multi-targeted tyrosine kinase inhibitors [32]. Therefore, blood pressure should be monitored in a constant position at each visit. In general, in subjects with known hypertension, antihypertensive treatment should be optimized prior to cabozantinib being started. In subjects with blood pressure $>150 \mathrm{mmHg}$ systolic or $>100 \mathrm{mmHg}$ diastolic, antihypertensive treatment should be further optimized by either a dose increase of existing medication and/or addition of further antihypertensive medication according to standard of care (e.g., treatment with angiotensin-converting enzyme inhibitors, angiotensin receptor blockers, and/or beta-blockers) [32].

\subsection{Fatigue}

Fatigue is a typical symptom of HCC and advanced liver cirrhosis, but is also a common $\mathrm{AE}$ of anti-angiogenic, multi-targeted tyrosine kinase inhibitors. Common causes of fatigue (e.g., anemia, depression and/or anxiety, nutrition, sleep disturbance, and hypothyroidism) should be ruled out or treated according to standard of care. In general, exercise might reduce side effects such as fatigue and therefore improve quality of life in patients with cancer; however, for HCC, this has not been shown. Due to the underlying liver cirrhosis, patients with HCC are often not fit enough for physical exercise, but whenever possible, light physical exercise (e.g., walking, cycling, or weight-bearing exercise) alternating with rest periods should be encouraged for patients under treatment with multi-targeted tyrosine kinase inhibitors [32].

\section{Ongoing Development of Cabozantinib in HCC}

Currently, several clinical trials with cabozantinib in patients with HCC are ongoing (clinicalTrials.gov, accessed 12 March 2020). The most advanced study is the COSMIC-312 trial ("Study of Cabozantinib in Combination With Atezolizumab Versus Sorafenib in Subjects With Advanced HCC Who Have Not Received Previous Systemic Anticancer Therapy"; ClinicalTrials.gov Identifier: NCT03755791). This is a phase 3 trial $(n=740)$ evaluating the safety and efficacy of cabozantinib in combination with the anti-PD-L1 antibody atezolizumab versus sorafenib as first-line systemic therapy in patients with advanced HCC. Additionally, a single-agent cabozantinib arm will be enrolled to determine this treatment's contribution to the overall safety and efficacy of the combination with atezolizumab (2:1:1 randomization). In the combination arm, the cabozantinib dosage is reduced to $40 \mathrm{mg}$ QD. In parallel, a multicenter, phase 1b trial ("Study of Cabozantinib in Combination With Atezolizumab to Subjects With Locally Advanced or Metastatic Solid Tumors"; ClinicalTrials.gov Identifier: NCT03170960) will assess safety, tolerability, preliminary efficacy, and pharmacokinetics of cabozantinib in combination with atezolizumab in different tumor entities, including HCC. This study is estimated to complete enrolment in December 2020.

Just recently, results of the multiple-cohort CheckMate040 phase 1/2 study ("An Immuno-therapy Study to Evaluate the Effectiveness, Safety and Tolerability of Nivolumab or Nivolumab in Combination With Other Agents in Patients With Advanced Liver Cancer"; ClinicalTrials.gov identifier: NCT01658878) reported the efficacy of two sorafenib-pretreated cabozantinib cohorts $(40 \mathrm{mg}$ QD) [40]. In the cabozantinib-nivolumab cohort $(n=36)$, the median PFS was 5.4 months, whereas it was 6.8 months in the cabozantinib-nivolumab-ipilimumab $(1 \mathrm{mg} / \mathrm{kg}$ every 6 weeks) cohort $(n=35)$. The median OS was 21.5 months in the doublet arm, whereas it was not reached in the triplet arm. Interestingly, a favorable outcome in an HCC patient with a RET amplification, a high tumor mutational burden (12.3 mutations per megabase), and PD-L1 expression of $5 \%$ was reported in a case report [41]. This patient achieved a PFS of more than 25 months with combination therapy of cabozantinib and nivolumab. Although, the frequency of RET amplifications in HCC is low (0.34\%), this warrants further investigations.

Additionally, cabozantinib (starting dosage $20 \mathrm{mg}$ QD) in combination with the anti-PD-L1 antibody durvalumab is being evaluated in a small, multi-gastrointestinal tumor cohort, phase $1 \mathrm{~b}$ trial (CAMILLA, $n=30$; ClinicalTrials. gov identifier: NCT03539822).

A neoadjuvant treatment with cabozantinib in combination with the anti-PD-1 antibody nivolumab is currently being studied in a single-arm, US, proof-of-concept, phase $1 \mathrm{~b}$ trial ["Feasibility and Efficacy of Neoadjuvant Cabozantinib Plus Nivolumab (CaboNivo) Followed by Definitive Resection for Patients With Locally Advanced Hepatocellular Carcinoma (HCC)," $n=15$; ClinicalTrials.gov identifier: NCT03299946]. In this study, patients will be treated for 8 weeks with cabozantinib (40 mg QD) in combination with nivolumab in the neoadjuvant setting.

Furthermore, a French, phase 4 trial ["Cabozantinib toLERANCE Study in HepatoCellular Carcinoma (CLERANCE)," $n=170$; ClinicalTrials.gov identifier: NCT03963206] is recruiting patients to further study the safety of cabozantinib in patients with advanced HCC previously treated with sorafenib and possibly another systemic treatment line. Several smaller phase 2 studies will evaluate 
the use of cabozantinib in special settings, e.g., in patients with HCC recurrence after liver transplantation (CaboTx: a Canadian, phase 2 study with cabozantinib to treat recurrent HCC post transplant, $n=20$; ClinicalTrials.gov identifier: NCT04204850, not yet recruiting). A Japanese phase 2 study has evaluated the safety and efficacy of cabozantinib in patients with advanced HCC sorafenib-pretreated or not in two different cohorts $(n=32$; ClinicalTrials.gov identifier: NCT03586973). The results of this trial are not reported yet.

Due to the negative studies of sorafenib as adjuvant treatment after resection or ablation or in combination with transarterial chemoembolization (TACE), so far no study activity of single-agent cabozantinib is being overseen in early- or intermediate-stage HCC patients [42, 43].

\section{Future Role of Cabozantinib in the Sequence of Systemic Treatment}

After FDA approval of atezolizumab and bevacizumab at the end of May 2020, sequencing of further systemic treatment in advanced HCC patients will become important but unpredictable. To date, it is unclear whether VEGF pathwaydirected tyrosine kinase inhibitors or ramucirumab or singleagent nivolumab or pembrolizumab will show activity after failure of atezolizumab and bevacizumab. Based on its broad approval status in HCC, sorafenib will be the only in-label systemic second-line treatment. Since MET is a promising target, especially in progressing HCC, a combined VEGF/ MET receptor inhibitor such as cabozantinib might be an interesting option after failure of atezolizumab and bevacizumab. However, this dilemma will only be resolved by appropriate clinical trials.

\section{Conclusion}

The MET, VEGF, and AXL receptor multi-targeted tyrosine kinase inhibitor cabozantinib is approved for patients with sorafenib-pretreated advanced HCC, but effective AE management is crucial to maximize the therapeutic benefit. Since atezolizumab and bevacizumab will be the preferred firstline systemic treatment for this disease very soon, sorafenib might become the standard second-line systemic treatment. As a further systemic treatment option, cabozantinib might be considered, since its use is independent from sorafenib tolerance or AFP levels. Further data are eagerly needed to guide clinicians on how to sequence targeted therapies after failure of immunotherapy in patients with advanced HCC.

Currently, cabozantinib is being further developed, especially in combination with immune checkpoint inhibitors, for different lines of HCC treatment.
Acknowledgements Open Access funding provided by Projekt DEAL.

\section{Compliance with Ethical Standards}

Funding No external funding was used in the preparation of this article.

Conflicts of interest Jörg Trojan has received research grants from Roche. He has received speaker and consulting honoraria from Amgen, Astra Zeneca, Bayer Healthcare, Bristol Myers-Squibb, Eisai, Ipsen, Merck Serono, Merck Sharp \& Dome, Lilly Imclone, and Roche.

Open Access This article is licensed under a Creative Commons Attribution-NonCommercial 4.0 International License, which permits any non-commercial use, sharing, adaptation, distribution and reproduction in any medium or format, as long as you give appropriate credit to the original author(s) and the source, provide a link to the Creative Commons licence, and indicate if changes were made. The images or other third party material in this article are included in the article's Creative Commons licence, unless indicated otherwise in a credit line to the material. If material is not included in the article's Creative Commons licence and your intended use is not permitted by statutory regulation or exceeds the permitted use, you will need to obtain permission directly from the copyright holder. To view a copy of this licence, visit http://creativecommons.org/licenses/by-nc/4.0/.

\section{References}

1. World Health Organization, News room, fact sheets, cancer. In: WHO [Internet]. 12 Sep 2018. http://www.who.int/news-room/ fact-sheets/detail/cancer.

2. European Association for the Study of the Liver. EASL clinical practice guidelines: management of hepatocellular carcinoma. J Hepatol. 2018;69(1):182-236.

3. Llovet JM, Ricci S, Mazzaferro V, et al. Sorafenib in advanced hepatocellular carcinoma. N Engl J Med. 2008;359(4):378-90.

4. Cheng AL, Kang YK, Chen Z, et al. Efficacy and safety of sorafenib in patients in the Asia-Pacific region with advanced hepatocellular carcinoma: a phase III randomised, double-blind, placebo-controlled trial. Lancet Oncol. 2009;10:25-34.

5. Bruix J, Qin S, Merle P, et al. Regorafenib for patients with hepatocellular carcinoma who progressed on sorafenib treatment (RESORCE): a randomised, double-blind, placebo-controlled, phase 3 trial. Lancet. 2017;389(10064):56-66.

6. Kudo M, Finn RS, Qin S, et al. Lenvatinib versus sorafenib in first-line treatment of patients with unresectable hepatocellular carcinoma: a randomised phase 3 non-inferiority trial. Lancet. 2018;391(10126):1163-73.

7. Zhu AX, Kang YK, Yen CJ, et al. Ramucirumab after sorafenib in patients with advanced hepatocellular carcinoma and increased $\alpha$-fetoprotein concentrations (REACH-2): a randomised, double-blind, placebo-controlled, phase 3 trial. Lancet Oncol. 2019;20(2):282-96

8. El-Khoueiry AB, Sangro B, Yau T, et al. Nivolumab in patients with advanced hepatocellular carcinoma (CheckMate 040): an open-label, non-comparative, phase 1/2 dose escalation and expansion trial. Lancet. 2017;389(10088):2492-502.

9. Zhu AX, Finn RS, Edeline J, et al. Pembrolizumab in patients with advanced hepatocellular carcinoma previously treated with sorafenib (KEYNOTE-224): a non-randomised, open-label phase 
2 trial [published correction appears in Lancet Oncol. 2018 Sep;19(9):e440]. Lancet Oncol. 2018;19(7):940-52.

10. Finn RS, Qin S, Ikeda M, et al. Atezolizumab plus bevacizumab in unresectable hepatocellular carcinoma. N Engl J Med. 2020;382(20):1894-905.

11. Zhou L, Liu XD, Sun M, et al. Targeting MET and AXL overcomes resistance to sunitinib therapy in renal cell carcinoma. Oncogene. 2016;35(21):2687-97.

12. Bouattour M, Raymond E, Qin S, et al. Recent developments of c-Met as a therapeutic target in hepatocellular carcinoma. Hepatology. 2018;67(3):1132-49.

13. Faivre S, Rimassa L, Finn RS. Molecular therapies for HCC: Looking outside the box. J Hepatol. 2020;72(2):342-52.

14. Garon EB, Heist RS, Seto T, et al. Capmatinib in METex14mutated (mut) advanced non-small cell lung cancer (NSCLC): Results from the phase II GEOMETRY mono-1 study, including efficacy in patients (pts) with brain metastases (BM). Presented at: 2020 American Association for Cancer Research Virtual Annual Meeting I: Virtual; April 27-28, 2020. Abstract CT082.

15. Rimassa L, Assenat E, Peck-Radosavljevic M, et al. Tivantinib for second-line treatment of MET-high, advanced hepatocellular carcinoma (METIV-HCC): a final analysis of a phase 3, randomised, placebo-controlled study. Lancet Oncol. 2018;19(5):682-93.

16. Abou-Alfa GK, Meyer T, Cheng AL, et al. Cabozantinib in Patients with Advanced and Progressing Hepatocellular Carcinoma. N Engl J Med. 2018;379(1):54-63.

17. Yakes FM, Chen J, Tan J, et al. Cabozantinib (XL184), a novel MET and VEGFR2 inhibitor, simultaneously suppresses metastasis, angiogenesis, and tumor growth. Mol Cancer Ther. 2011;10(12):2298-308.

18. Matsui J, Yamamoto Y, Funahashi Y, et al. E7080, a novel inhibitor that targets multiple kinases, has potent antitumor activities against stem cell factor producing human small cell lung cancer H146, based on angiogenesis inhibition. Int J Cancer. 2008;122(3):664-71.

19. Wilhelm SM, Carter C, Tang L, et al. BAY 43-9006 exhibits broad spectrum oral antitumor activity and targets the RAF/MEK/ERK pathway and receptor tyrosine kinases involved in tumor progression and angiogenesis. Cancer Res. 2004;64(19):7099-109.

20. Wilhelm SM, Dumas J, Adnane L, et al. Regorafenib (BAY 73-4506): a new oral multikinase inhibitor of angiogenic, stromal and oncogenic receptor tyrosine kinases with potent preclinical antitumor activity. Int J Cancer. 2011;129(1):245-55.

21. Exelixis Inc. Cabometyx ${ }^{\circledR}$ (cabozantinib) tablets for oral use: US prescribing information. 2019. https://cabometyx.com/resources. Accessed 26 Feb 2020.

22. Ipsen Pharma. Cabometyx film-coated tablets: EU summary of product characteristics. 2019. https://www.ema.europa.eu. Accessed 26 Feb 2020.

23. Firtina Karagonlar Z, Koc D, Iscan E, Erdal E, Atabey N. Elevated hepatocyte growth factor expression as an autocrine c-Met activation mechanism in acquired resistance to sorafenib in hepatocellular carcinoma cells. Cancer Sci. 2016;107(4):407-16.

24. Rimassa L, Abbadessa G, Personeni N, et al. Tumor and circulating biomarkers in patients with second-line hepatocellular carcinoma from the randomized phase II study with tivantinib. Oncotarget. 2016;7(45):72622-33.

25. Schöffski P, Gordon M, Smith DC, et al. Phase II randomised discontinuation trial of cabozantinib in patients with advanced solid tumours. Eur J Cancer. 2017;86:296-304.

26. Kelley RK, Verslype C, Cohn AL, et al. Cabozantinib in hepatocellular carcinoma: results of a phase 2 placebo-controlled randomized discontinuation study. Ann Oncol. 2017;28(3):528-34.

27. Johnson PJ, Berhane S, Kagebayashi C, et al. Assessment of liver function in patients with hepatocellular carcinoma: a new evidence-based approach-the ALBI grade. J Clin Oncol. 2015;33(6):550-8.

28. Miksad R, Cicin I, Chen Y. Outcomes based on albumin-bilirubin (ALBI) grade in the phase 3 CELESTIAL trial of cabozantinib versus placebo in patients with advanced hepatocellular carcinoma (HCC). Ann Oncol. 2019;30(Suppl 4):iv134.

29. Fan SY, Eiser C, Ho MC. Health-related quality of life in patients with hepatocellular carcinoma: a systematic review. Clin Gastroenterol Hepatol. 2010;8(7):559-64.

30. Abou-Alfa GK, Mollon P, Meyer T, et al. Quality-adjusted life years assessment using cabozantinib for patients with advanced hepatocellular carcinoma (aHCC) in the CELESTIAL trial. J Clin Oncol. 2019;37(4_suppl):207.

31. Finkelmeier F, Scheiner B, Leyh C, et al. Cabozantinib in advanced hepatocellular carcinoma: Efficacy and safety data from an international multicenter real-world cohort. J Clin Oncol. 2020;38(15_suppl):e16668.

32. Rimassa L, Danesi R, Pressiani T, Merle P. Management of adverse events associated with tyrosine kinase inhibitors: improving outcomes for patients with hepatocellular carcinoma. Cancer Treat Rev. 2019;77:20-8.

33. Lacouture ME, Reilly LM, Gerami P, Guitart J. Hand foot skin reaction in cancer patients treated with the multikinase inhibitors sorafenib and sunitinib. Ann Oncol. 2008;19:1955-61.

34. Vaklavas C, Lenihan D, Kurzrock R, Tsimberidou AM. Anti-vascular endothelial growth factor therapies and cardiovascular toxicity: what are the important clinical markers to target? Oncologist. 2010;15:130-41.

35. Sanders KM, Koh SD, Ward SM. Interstitial cells of cajal as pacemakers in the gastrointestinal tract. Annu Rev Physiol. 2006;68:307-43.

36. Wang D, Lehman RE, Donner DB, Matli MR, Warren RS, Welton ML. Expression and endocytosis of VEGF and its receptors in human colonic vascular endothelial cells. Am J Physiol Gastrointest Liver Physiol. 2002;282:G1088-96.

37. Abou-Alfa GK, Meyer T, Cheng AL, et al. Association of adverse events (AEs) with efficacy outcomes for cabozantinib (C) in patients (pts) with advanced hepatocellular carcinoma (aHCC) in the phase III CELESTIAL trial. J Clin Oncol. 2019;37(15_suppl):4088.

38. Schmidinger M, Danesi R. Management of adverse events associated with cabozantinib therapy in renal cell carcinoma. Oncologist. 2018;23(3):306-15.

39. Nguyen L, Holland J, Mamelok R, et al. Evaluation of the effect of food and gastric $\mathrm{pH}$ on the single-dose pharmacokinetics of cabozantinib in healthy adult subjects. J Clin Pharmacol. 2015;55(11):1293-302.

40. Yau T, Zagonel V, Santoro A, et al. Nivolumab (NIVO) + ipilimumab (IPI) + cabozantinib (CABO) combination therapy in patients (pts) with advanced hepatocellular carcinoma (aHCC): results from CheckMate 040. J Clin Oncol. 2020;38(Suppl 4):abstr 478.

41. Yang X, Shi J, Chen X, Jiang Y, Zhao H. Efficacy of Cabozantinib and Nivolumab in Treating Hepatocellular Carcinoma with RET Amplification, High Tumor Mutational Burden, and PD-L1 Expression. Oncologist. 2020;25(6):470-4.

42. Bruix J, Takayama T, Mazzaferro V, et al. Adjuvant sorafenib for hepatocellular carcinoma after resection or ablation (STORM): a phase 3, randomised, double-blind, placebo-controlled trial. Lancet Oncol. 2015;16(13):1344-54.

43. Lencioni R, Llovet JM, Han G, et al. Sorafenib or placebo plus TACE with doxorubicin-eluting beads for intermediate stage HCC: the SPACE trial. J Hepatol. 2016;64(5):1090-8. 